\title{
Estimation of the Incremental Cumulative Cost of HIV Compared with a Non-HIV Population
}

\author{
Joshua P. Cohen ${ }^{1} \cdot$ Anne Beaubrun ${ }^{2} \cdot$ Yao Ding ${ }^{3} \cdot$ Rolin L. Wade $^{3} \cdot$ Dionne M. Hines $^{3}$
}

Published online: 26 March 2020

(c) The Author(s) 2020

\begin{abstract}
Objective There are limited real-world data comparing cumulative incremental healthcare costs in people living with HIV (PLWH) and those without HIV. This study evaluated all-cause cumulative and incremental costs in PLWH in the US using a matched-cohort design.

Materials and Methods This retrospective, multi-year, cross-sectional analysis evaluated annual costs from 2013 to 2017 , and projected cumulative costs of HIV from age 25 to 69 years. IQVIA's commercial adjudicated claims database was used to identify patients with HIV and match them with patients without HIV (controls). Cumulative all-cause costs were derived from the health plan-allowed costs incurred from ages 25-69 years. Undiscounted, discounted, and incremental costs between PLWH and non-HIV populations were reported in 2017 US dollars (US\$), and annual all-cause costs were estimated for each year by 10 -year age bands.

Results A total of 25,261, 24,134, 31,654, 35,374, and 29,039 PLWH and 75,783, 72,402, 94,962, 106,122, and 87,117 matched controls were identified in the years 2013 through 2017, respectively. The mean undiscounted cumulative costs were $\$ 1,840,554$ for PLWH and $\$ 285,065$ for controls, an incremental cost difference of $\$ 1,555,489$, while the mean discounted cumulative cost for PLWH was $\$ 983,897$ compared with $\$ 133,340$ for controls, an incremental cost difference of $\$ 850,557$. Mean all-cause annual and cumulative costs were up to seven times higher for PLWH compared with controls. There was a trend for costs to increase each year with increasing age.

Limitations and Conclusions While cumulative all-cause cost estimates only approximate total cost burden for any given patient, and the results of this study may not be generalizable to all population subgroups, this is one of the first US studies to examine annual and cumulative costs in a real-world cohort of commercially insured PLWH compared with a population without HIV. In this large, representative sample of commercially insured US adults with HIV, PLWH had substantially higher all-cause cumulative costs than individuals without HIV.
\end{abstract}

Contents of this manuscript have been previously presented at HIV DART and Emerging Viruses 2018, 27-29 November, Miami, FL, USA, and the American Pharmacists Association (APhA) Annual Meeting and Exposition 2019, 22-25 March, Seattle, WA, USA.

Electronic supplementary material The online version of this article (https://doi.org/10.1007/s41669-020-00209-8) contains supplementary material, which is available to authorized users.

Dionne M. Hines

dionne.hines@iqvia.com

1 Center for the Study of Drug Development, Tufts University, Boston, MA, USA

2 Division of Health Economics and Outcomes Research, Gilead Sciences, Foster City, CA, USA

3 Department of Health Economics and Outcomes Research, Real-World Evidence, IQVIA, One IMS Drive, Plymouth Meeting, PA 19462, USA

\section{Key Points for Decision Makers}

Mean all-cause annual and cumulative costs are up to seven times higher in patients with HIV, compared with a non-infected population.

Costs among patients with HIV increase with increasing age.

\section{Introduction}

In the US, an estimated 1.1 million people were living with HIV in 2016 and approximately 39,000 individuals were newly diagnosed in 2017 [1]. Although the incidence of HIV has steadily declined over the past 10 years [2-4], 
it remains a serious public health concern given the economic and social burden.

The economic burden of HIV has been measured via different approaches. Among 342,732 patients with HIV or AIDS in the Medical Expenditure Panel Survey from 2002 to 2011, adjusted average costs were approximately $\$ 10.7$ billion higher than the costs for those without HIV/ AIDS [5]. HIV healthcare costs are largely driven by pharmacy utilization among general HIV populations [6, 7], whereas patients who present with advanced disease have substantially higher costs, primarily driven by inpatient care $[8,9]$. A prior US study reported that the lifetime cost of HIV for a patient infected at age 35 years was $\$ 326,500$ in 2012 US dollars (US\$), with $60 \%$ of costs attributed to antiretroviral (ARV) medications, $15 \%$ to other medications, and 25\% to non-drug costs [8]. However, this study relied on dated lifetime cost estimates and was based on a simulated model of HIV. The cited studies emphasize the need for prevention, early detection of HIV, and timely initiation of ARV therapy (ART).

Although existing studies and models have established the high economic burden in patients with HIV annually or over a relatively longer timeframe (e.g. 10 years), there are limited real-world data on the comparison of cumulative healthcare costs in people living with HIV (PLWH) and individuals without HIV. Hence, the objectives of this study were to (1) evaluate all-cause cumulative costs in PLWH from ages 25-69 years based on real-world US claims data; and (2) estimate the incremental cost of HIV using a matched-cohort design comparing HIV and nonHIV individuals.

\section{Methods}

\subsection{Study Design and Data Source}

This study was a retrospective, multi-year, cross-sectional, case-control analysis evaluating annual costs across a 5-year period (2013-2017) using a commercial US realworld adjudicated claims database (IQVIA's PharMetrics Plus $[\mathrm{P}+]) . \mathrm{P}+$ is a closed database of medical and pharmacy claims data of over 150 million unique individuals including national and subnational health plans as well as self-insured employer groups. The database has detailed information on inpatient/outpatient diagnoses and procedures documented by primary care and specialty providers, as well as pharmacy utilization. The health plan cost information is complete in the database and is representative of the national, commercially insured population for individuals aged $\leq 65$ years. Working adults older than 65 years of age, as well as those purchasing supplemental commercial Medicare insurance, are also included in the database, albeit at smaller sample sizes. The cost data from the database used in this study was the allowed amount for payment as determined by the health plan, which included both the actual amount paid by the plan as well as the patient responsibility, or out-of-pocket cost. All data are compliant with the Health Insurance Portability and Accountability Act to protect patients' privacy.

\subsection{Patient Selection}

Adult ( $\geq 18$ years of age) PLWH were identified from 1 January 2013 through 30 September 2017 (selection window) and were evaluated on an annual basis. PLWH were required to meet the following study criteria: (1) one or more diagnoses of HIV-1 (International Classification of Diseases, Ninth Revision [ICD-9] 042, V08; ICD, Tenth Revision [ICD-10] $079.53, \mathrm{Z21})$ on any non-ancillary medical claim during the respective evaluation (calendar) year; (2) one or more claims for any ARV during the respective evaluation year; and (3) continuous health plan enrollment with both medical and pharmacy benefits throughout the respective evaluation year. Patients with missing information on age, sex, prescription, or diagnosis were excluded.

PLWH were directly matched with non-HIV patients (no evidence of HIV diagnosis or ART use during the entire study period) on age, sex, geographic region, and index year at a 1:3 ratio within each evaluation year (five separate annual matched cohorts). Matched controls were required to meet the same health plan enrollment criteria. Data included in this study were from 1 January 2012 through 30 September 2017 (study window) to allow for confirmation of non-HIV status.

\subsection{Measures}

\subsubsection{Patient Characteristics}

Demographics (age, sex, geographic region, payer type, and health plan type) were assessed within each evaluation year and compared between PLWH and matched non-HIV patients. Measures were based on the first claim within the respective year. Standard comorbidities and neuropsychiatric conditions were also evaluated, based on the observation of one or more claims with a diagnosis for the respective condition.

\subsubsection{Cumulative Costs}

Cumulative costs were defined as direct healthcare costs (medical and pharmacy) associated with treating HIV patients from the age of 25-69 years, and were evaluated from age 25 years, based on reports from the Centers for 
Disease Control and Prevention that HIV infections peak around this age [10]. A bootstrapping approach was applied to the estimation of cumulative costs (described in the Statistical Analysis section), wherein cost calculations were based on costs per age using cost data over a 5-year period (2013-2017). Costs generated for each age between ages 25 and 69 years were summed to estimate costs over a 44-year period. For the cost estimates, undiscounted, discounted (3\% annual rate applied to discount future costs to net present value as in Eq. 1), and incremental costs between matched HIV and non-HIV populations were reported in 2017 US\$ via the standard calculation for the present value:

Future value (discounted annual cost)

$$
=\frac{\text { Future value (undiscounted annual cost) }}{(1+\text { annual discount rate of } 3 \%)^{\text {Number of years }}}
$$

\subsubsection{Annual Costs}

A cross-sectional evaluation of annual healthcare costs was performed for each year between 2013 and 2017. All-cause costs were estimated by 10 -year age bands for PLWH and non-HIV enrollees, and included costs associated with inpatient, outpatient, emergency department (ED), and pharmacy services utilized within each evaluation year. So that each age band contained 10 equal years of data, costs were estimated for patients beginning at 20 years of age and ending at 69 years of age (data in $\mathrm{P}+$ are limited for older patients, thus estimates for patients beyond this point may not be representative of the true population). Costs were reported as mean, standard deviation (SD), median, first quartile, and third quartile costs across the respective age bands (20-29, 30-39, 40-49, 50-59, 60-69 years). Upon calculating the per year cost for each age band, the average total cost across the years was estimated. The patient's age as of 2017 was used to determine the age band for which their costs were included for reporting the average total cost.

For the year 2017, data were not available beyond September at the time of the analysis, thus annual costs in 2017 were estimated using Eq. 2:

Estimated annual cost in 2017

$$
=(\text { cost in } 2017 Q 1 \text { to } Q 3) \times(1+\text { average ratio })
$$

where average ratio was calculated by the costs in the fourth quarter of years 2013-2016 divided by the costs in the first three quarters in 2013-2016; for example, where (Eq. 3):

Cost ratio for $2013=\frac{Q 4 \text { cost in } 2013}{Q 1 \text { to } Q 3 \operatorname{cost} \text { in } 2013}$
In addition to reporting annual costs by age band, costs were also reported annually by place of service (inpatient, outpatient, ED, and pharmacy).

Cumulative and annual costs were expressed as the health plan allowed amount (amount paid by the health plan + patients' co-pay amount). All costs were adjusted to 2017 US\$ using the Bureau of Labor Statistics Consumer Price Index for medical care [11].

\subsection{Statistical Analyses}

All analyses were performed using SAS version 9.3 (SAS Institute, Inc., Cary, NC, USA). Differences in baseline characteristics between PLWH and matched cohorts were tested using the Wilcoxon signed-rank test for continuous measures and McNemar's test for categorical measures. Descriptive statistics were used to report all-cause healthcare costs across age bands by each calendar year, and for reporting costs by place of service (mean, $\mathrm{SD}$, median). A $p$ value $<0.05$ indicated a significant difference in costs. Cumulative costs were estimated using a bootstrapping approach to generate mean and median costs and 2.5th and 97.5th confidence intervals (CIs) for patients sampled at each age from 25 to 69 years. The bootstrapping approach allowed for us to account for the skewness typically observed in cost data by resampling the population in generating the cost data, thereby minimizing bias and reducing the standard errors in the estimates. Bootstrapping with 1000 independent replications for each age band in PLWH and non-HIV patients was performed [12]. When evaluating the number of patients available for sampling at each age, we observed that the smallest sample was for patients aged 69 years $(n=256)$. Based on this count, 250 patients were randomly sampled for each bootstrapping replicate in the base-case scenario. To evaluate the impact of the number of patients sampled on the cost estimate, two sensitivity analyses were performed. In the first sensitivity analysis, we used a 50\% random sample of patients in each age, and, in a separate analysis, the full population for the respective ages was used as the number of patients sampled for each bootstrapping replication.

\section{Results}

A total of $25,261,24,134,31,654,35,374$, and $29,039 \mathrm{HIV}$ cases and 75,783, 72,402, 94,962, 106,122, and 87,117 matched controls were identified from 2013 through 2017, respectively (Table 1). In both cases and controls, mean (SD) age was 47 (11) years, and the majority were male (80-82\%) and covered by commercial/self-insured health plans 
throughout each evaluation year. The majority lived in the Southern US (59\%), while fewer came from other regions (Northeast, 16\%; Midwest, 17\%; West, 8\%) (electronic supplementary Table 1). Despite matching, all comorbidities of interest (except for rheumatologic disease) were significantly more prevalent among PLWH compared with their controls. Noteworthy differences were observed between PLWH and non-HIV patients with respect to alcohol/drug abuse (year 2017 data: $14.3 \%$ vs. $5.7 \%$, respectively; $p<0.0001$ ), depression ( $14.3 \%$ vs. $5.2 \%$, respectively; $p<0.0001)$, dyslipidemia (36.0\% vs. $22.5 \%$, respectively; $p<0.0001$ ), and hypertension $(31.1 \%$ vs. $23.7 \%$; $p<0.0001)$ (Table 2$)$.

\subsection{Cumulative Cost Estimation}

Base-case results for the cumulative cost estimation are presented in Table 3 . The mean undiscounted cumulative cost for PLWH was $\$ 1,840,554$ (median: $\$ 1,838,889$; 95\% CI $\$ 1,792,559-\$ 1,894,414)$. For non-HIV enrollees, the mean undiscounted cumulative cost was $\$ 285,065$ (median \$284,324; 95\% CI \$265,664-\$307,809), with an incremental mean cost difference of $\$ 1,555,489$ (median $\$ 1,554,030 ; 95 \%$ CI $\$ 1,501,464-\$ 1,612,220)$. The mean discounted cumulative cost for PLWH was $\$ 983,897$ (median $\$ 982,369 ; 95 \%$ CI $\$ 958,424-\$ 1,013,820$ ), whereas the mean discounted cumulative cost for non-HIV enrollees was $\$ 133,340$ (median $\$ 133,056$; $95 \%$ CI $\$ 123,317-\$ 145,604$ ), with an incremental mean cost difference of $\$ 850,557$ (median \$849,726; 95\% CI \$821,541-\$881,970). Sensitivity analyses of cumulative costs appeared robust and consistent with the base-case lifetime cost estimates (electronic supplementary Table 2).

\subsection{Annual Cost Estimation}

Age-based analyses revealed a general trend toward increasing costs from year-to-year and with increasing age band. This was more notable among PLWH. Additionally, costs incurred during each year were higher among patients in the older age bands. For instance, in 2017, median costs for PLWH were $\$ 32,053$ for those aged $20-29$ years, $\$ 34,194$ for those aged $30-39$ years, $\$ 35,167$ for those aged $40-49$ years, $\$ 36,623$ for those aged $50-59$ years, and $\$ 38,325$ for those aged 60-69 years (electronic supplementary Table 3).

For each year of evaluation, mean annual all-cause costs were consistently nearly seven times higher for PLWH compared with non-HIV patients. For PLWH, the mean (SD) total healthcare cost across the years was $\$ 40,352(\$ 51,562)$. The majority of costs (72.9\%) was attributed to pharmacy utilization [ $\$ 29,434(\$ 21,495)]$, followed by outpatient visits

Table 1 Sample selection by evaluation year

\begin{tabular}{|c|c|c|c|c|c|c|}
\hline Step & Attrition criteria & 2013 & 2014 & 2015 & 2016 & 2017 \\
\hline \multicolumn{7}{|c|}{ HIV Cohort } \\
\hline 1 & $\begin{array}{l}\text { Patients with a diagnosis of HIV1 on one or more non- } \\
\text { ancillary claims during the evaluation year }{ }^{\mathrm{a}}\end{array}$ & 47,223 & 57,634 & 66,369 & 67,859 & 56,558 \\
\hline 2 & $\begin{array}{l}\text { At least one claim for any } \mathrm{ART}^{\mathrm{b}} \text { within the respective evalu- } \\
\text { ation year }\end{array}$ & 36,536 & 43,127 & 51,502 & 53,170 & 46,293 \\
\hline 3 & $\begin{array}{l}\text { Continuous health plan enrollment with both medical and } \\
\text { pharmacy benefits }\end{array}$ & 25,557 & 24,412 & 31,994 & 35,740 & 29,194 \\
\hline 4 & $\begin{array}{l}\text { Patients aged } \geq 18 \text { years as of the index year and without } \\
\text { data quality issues (missing age, sex, prescription, or } \\
\text { diagnosis information) }\end{array}$ & 25,261 & 24,134 & 31,654 & 35,374 & 29,039 \\
\hline \multicolumn{7}{|c|}{ Non-HIV cohort } \\
\hline 1 & $\begin{array}{l}\text { Patients without a diagnosis of HIV or } \mathrm{ART}^{\mathrm{b}} \text { anytime dur- } \\
\text { ing the study period }\end{array}$ & $6,330,887$ & $6,330,887$ & $6,330,887$ & $6,330,887$ & $6,330,887$ \\
\hline 2 & $\begin{array}{l}\text { Continuous health plan enrollment with both medical and } \\
\text { pharmacy benefits throughout the year }\end{array}$ & $1,398,706$ & $1,284,434$ & $1,414,770$ & $1,442,874$ & $1,126,406$ \\
\hline 3 & $\begin{array}{l}\text { Patients aged } \geq 18 \text { years and without data quality issues } \\
\text { (missing age, sex, prescription, or diagnosis information) }\end{array}$ & $1,037,275$ & 950,833 & $1,055,887$ & $1,076,973$ & 904,970 \\
\hline \multicolumn{7}{|c|}{ Matched sample } \\
\hline \multicolumn{2}{|c|}{ HIV patients remaining after $1: 3$ direct match } & 25,261 & 24,134 & 31,654 & 35,374 & 29,039 \\
\hline \multicolumn{2}{|c|}{ Non-HIV patients remaining after $1: 3$ direct match } & 75,783 & 72,402 & 94,962 & 106,122 & 87,117 \\
\hline
\end{tabular}

ART antiretroviral therapy, HIV human immunodeficiency virus, NNRTIs non-nucleoside reverse transcriptase inhibitors, NRTIs nucleoside reverse transcriptase inhibitors, PIs protease inhibitors, INSTIs integrase strand transfer inhibitors

${ }^{a}$ For the year 2017, the end of the study period (30 September 2017) was the end of evaluation

${ }^{\mathrm{b}}$ ART included the following drug classes: NNRTIs, NRTIs, PIs, fusion inhibitors, entry inhibitors, and INSTIs

${ }^{\mathrm{c}}$ Matching variables included age as of evaluation year, sex, and geographic region 


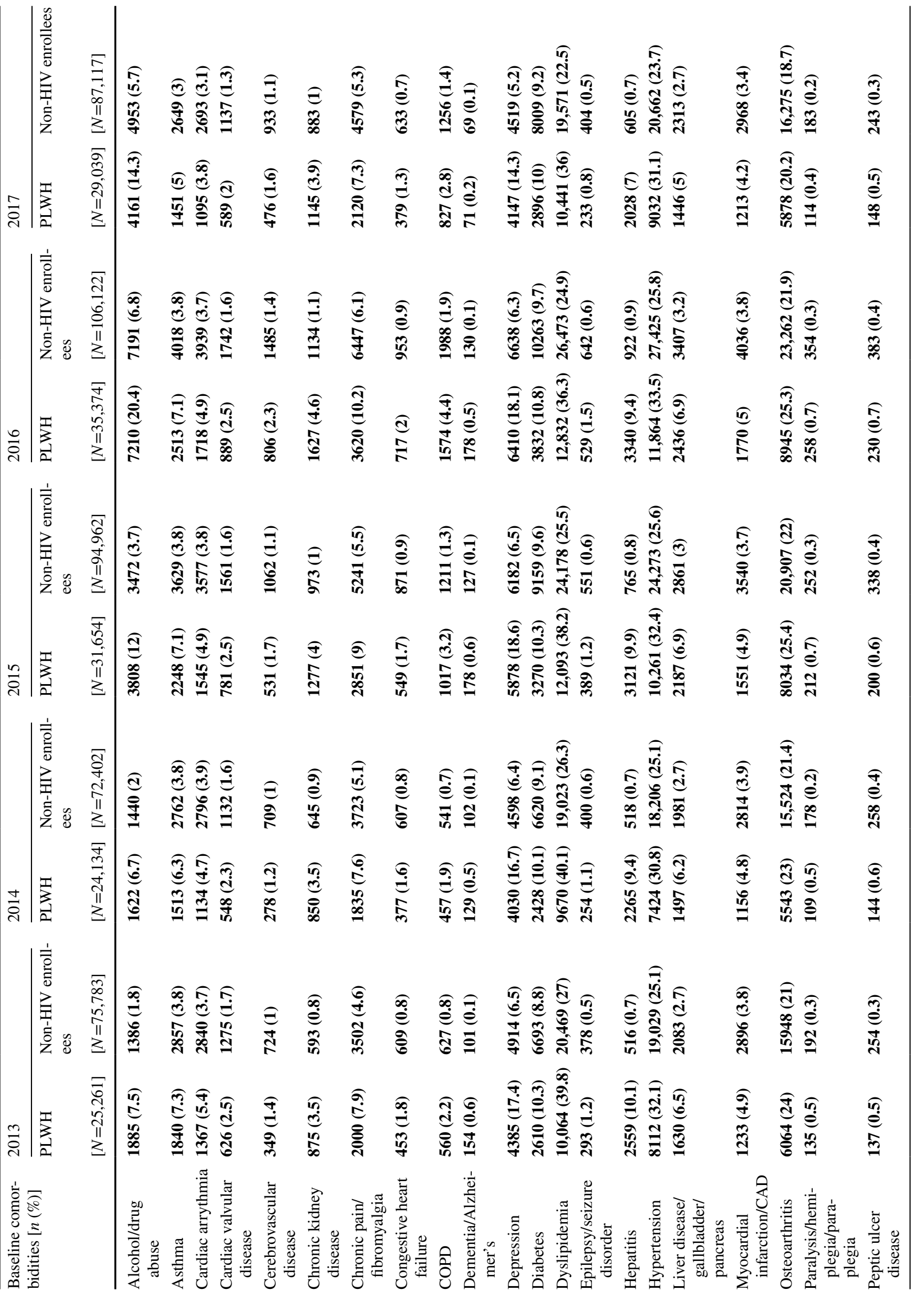




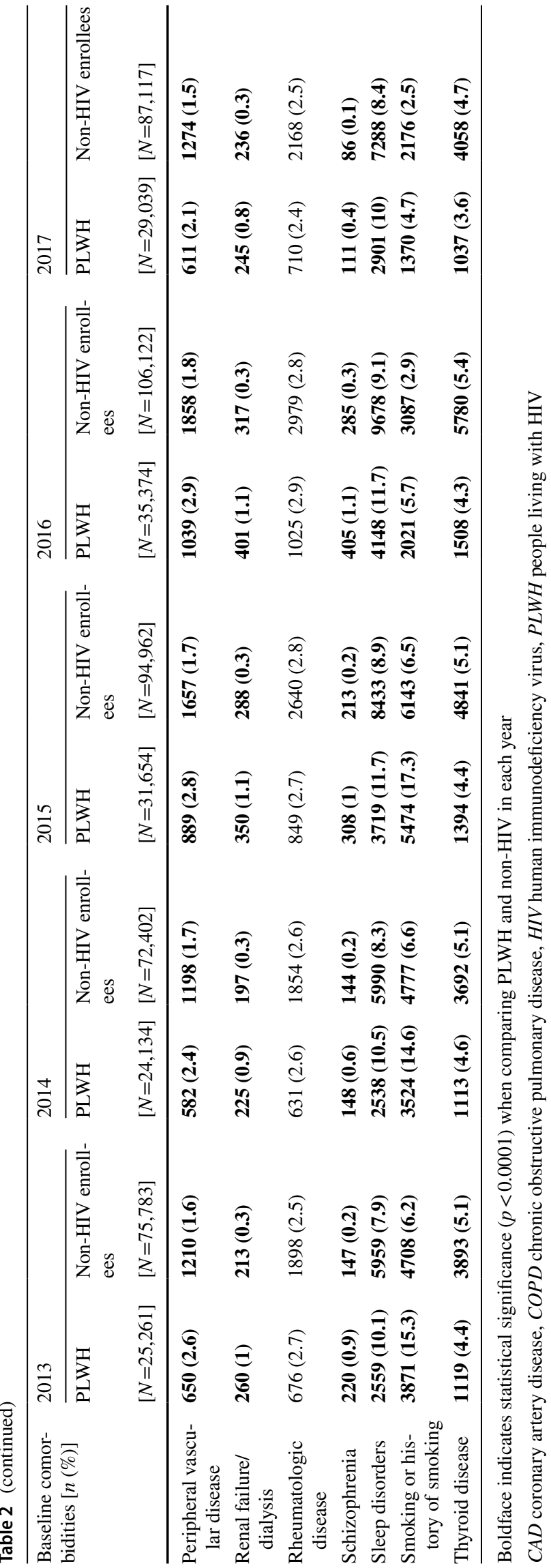

[\$6338 (\$34,334)] and hospitalization [\$4032 (\$25,401)]. For non-HIV patients, approximately half of the costs $(51.2 \%)$ were attributed to outpatient visits [\$3123 $(\$ 22,490)]$, followed by hospitalization [ $\$ 1380(\$ 13,794)$ ] and pharmacy utilization [\$1344 (\$7724)] (Table 4).

\section{Discussion}

To our knowledge, this is the first US study using a retrospective cohort study design to compare incremental cumulative healthcare costs in commercially insured PLWH and patients without HIV between the ages of 25 and 69 years. In this study, mean all-cause annual healthcare costs from ages 25-69 were almost seven times higher in PLWH compared with individuals without HIV, with a mean undiscounted incremental amount of more than $\$ 1.5$ million and an average discounted incremental cost of more than $\$ 850,000$ in cumulative costs. This finding was further supported by sensitivity analyses.

Evidence regarding the cumulative costs in PLWH is limited. Prior research on lifetime cost estimates used computer simulation models with model inputs derived from the public domain. For example, a previous analysis conducted by Schackman et al. used a simulated cost model and projected costs to estimate that the mean discounted lifetime cost of HIV for a patient infected at age 35 years was $\$ 326,500$ in 2012 US\$ [7]. In another model-based study, Farnham et al. found lifetime cost estimates of $\$ 332,300$ and \$443,000 (2012 US\$) among US individuals entering care with CD4 201-350/ $\mu \mathrm{L}$ and $>500 / \mu \mathrm{L}$, respectively [13]. As advancements in the HIV care model have been made since these reports, the cost inputs used to derive the estimates presented in these studies are now dated. The current study utilized more contemporary data based on real-world health insurance claims to report actual medical and pharmacy costs incurred, as dictated by the health plan reimbursement model. Our evaluation yielded cost estimates that were two to three times higher than those previously reported. In addition to the use of non-simulated cost data, our approach differed from the previous studies in that costs were estimated from the age of 25 years, which represents the age at which infection rates peak [10], up to the age of 69 years (i.e. over a 44-year span), whereas Schackman et al. estimated costs assuming 29.5 years with HIV [8] and Farnham et al. estimated 38 years with HIV [13]. Additional factors potentially contributing to the discrepancies in cost estimates might relate to the costs included in each analysis. For example, Schackman et al. note that ED, inpatient, and non-HIV clinic visits may not have been adequately captured because HIV clinics were the source of the data [8]. In using healthcare claims for our analysis, we were able to capture all costs associated with medical and pharmacy services 
Table 3 Base-case results for cumulative costs in matched PLWH and non-HIV enrollees

\begin{tabular}{|c|c|c|c|c|c|c|}
\hline \multirow{2}{*}{$\begin{array}{l}\text { All-cause health- } \\
\text { care costs from ages } \\
25-69 \text { years }\end{array}$} & \multicolumn{3}{|c|}{ Undiscounted costs } & \multicolumn{3}{|c|}{ Discounted costs } \\
\hline & HIV & Non-HIV & $\begin{array}{l}\text { Incremental HIV vs. } \\
\text { non-HIV }\end{array}$ & HIV & Non-HIV & $\begin{array}{l}\text { Incremental HIV vs. } \\
\text { non-HIV }\end{array}$ \\
\hline Mean & $\$ 1,840,554$ & $\$ 285,065$ & $+\$ 1,555,489$ & $\$ 983,897$ & $\$ 133,340$ & $+\$ 850,557$ \\
\hline Median & $\$ 1,838,889$ & $\$ 284,324$ & $+\$ 1,554,030$ & $\$ 982,369$ & $\$ 133,056$ & $+\$ 849,726$ \\
\hline First quartile & $\$ 1,822,962$ & $\$ 277,635$ & $+\$ 1,536,956$ & $\$ 975,040$ & $\$ 129,529$ & $+\$ 840,228$ \\
\hline Third quartile & $\$ 1,857,624$ & $\$ 291,705$ & $+\$ 1,574,712$ & $\$ 992,677$ & $\$ 136,299$ & $+\$ 860,746$ \\
\hline $\begin{array}{l}95 \% \text { CI (2.5th and } \\
97.5 \text { th percentiles) }\end{array}$ & $\begin{array}{r}(\$ 1,792,559- \\
\$ 1,894,414)\end{array}$ & $\begin{array}{r}(\$ 265,664- \\
\$ 307,809)\end{array}$ & $\begin{array}{c}+(\$ 1,501,464- \\
\$ 1,612,220)\end{array}$ & $\begin{array}{l}(\$ 958,424- \\
\$ 1,013,820)\end{array}$ & $\begin{array}{r}(\$ 123,317- \\
\$ 145,604)\end{array}$ & $\begin{array}{c}+(\$ 821,541- \\
\$ 881,970)\end{array}$ \\
\hline
\end{tabular}

Bootstrapping approach using 1000 replications with 250 patients randomly sampled for each replicate

$H I V$ human immunodeficiency virus, $P L W H$ people living with HIV, CI confidence interval

covered by the insurance plan. It is worth noting that costs captured in this study are based on the actual payments made by health plans to medical providers and pharmacies, and include patient out-of-pocket costs, which may differ from rates derived from fee schedules, cost-to-charge ratios, other payers, or treatment estimates based on average wholesale price (AWP).

Over the 5-year period, annual mean all-cause costs were almost seven times higher in PLWH compared with nonHIV enrollees. Consistent with previous cost estimates using US claims data [14], we identified pharmacy utilization as the major contributor to total annual all-cause costs among PLWH, largely due to the high cost of ART. A subanalysis of costs incurred in 2017 revealed that ART accounts for more than $85 \%$ of pharmacy costs, and that these costs increase as patients age (electronic supplementary Table 4).

As ART has improved and life expectancy has increased, the proportion of adults over 50 years of age living with HIV has increased [15]. Consequently, the proportion of PLWH with comorbidities has increased over time, across both age groups and different payer types (commercial insurance, Medicare, Medicaid) in the US [16-18]. Improving care and quality of life for PLWH is crucial, and greater support is necessary for preventive measures to avert the transmission of the disease. Studies show that the early initiation of ART and treatment with pre-exposure prophylaxis can be cost-effective measures to control and even avoid long-term costs associated with HIV $[19,20]$.

This study expands upon previous estimations of lifetime costs for HIV by providing contemporary estimates of cumulative cost of HIV from age 25-69 years using recent real-world data generated from HIV patient claims, compared with matched uninfected counterparts. This study is strengthened by the inclusion of a representative population of HIV patients across the US, the use of actual rather than projected simulated costs, and the inclusion of a 5-year annual cost assessment that allows for the evaluation of year-to-year variations. In addition, sensitivity analyses were performed to substantiate the estimates.

\subsection{Limitations}

This study was limited by several factors. The cumulative all-cause cost estimate from ages 25 to 69 years only approximates total cost burden. Patients over 69 years of age are not captured and may experience costs different than those evaluated, as might patients who contract HIV later than 25 years of age or die before 69 years of age. Costs reported in this study were not evaluated within specific risk groups, and cumulative costs may be higher or lower among certain subpopulations. Administrative claims data are used primarily for billing and reimbursement purposes, and certain clinical conditions may potentially be under- or overcoded as a result of coding conventions designed around reimbursement considerations, leading to misclassification bias. Lastly, IQVIA's Real-World Data Adjudicated Claims Database-US (PharMetrics Plus) is representative of the US commercially insured population but may not be generalizable to all population groups, such as patients covered by Medicare and Medicaid.

\section{Conclusions}

Cumulative healthcare costs from ages 25 to 69 years were almost seven times higher in PLWH than in individuals without HIV. The cost of managing HIV is substantial and burdensome to patients and the healthcare system. Current cost estimates should be used when evaluating the economic impact of treating those with HIV and when developing strategies to prevent HIV infection, or to estimate the cost effectiveness of HIV prevention activities. To improve patient outcomes and reduce the economic burden of HIV, 


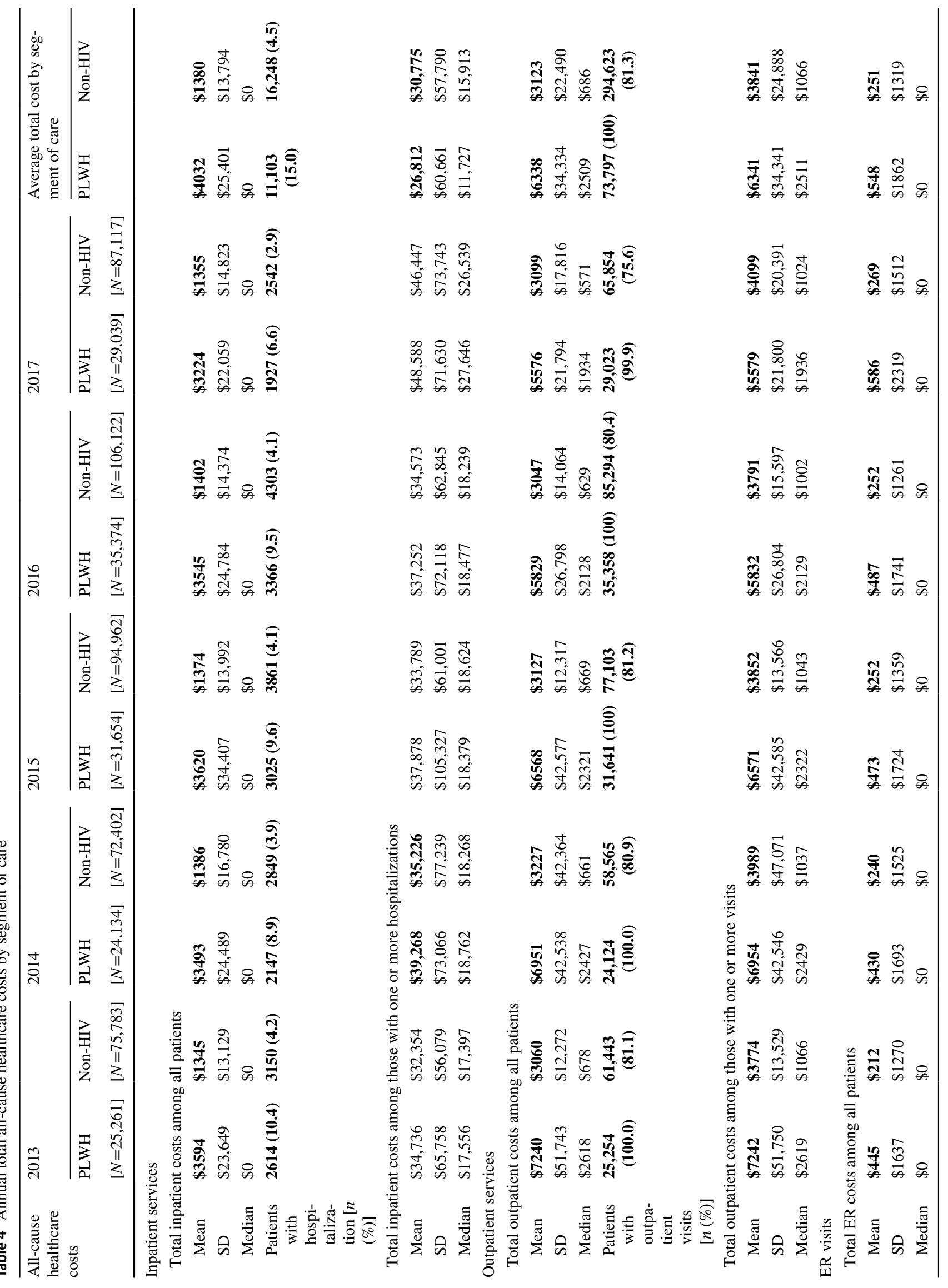




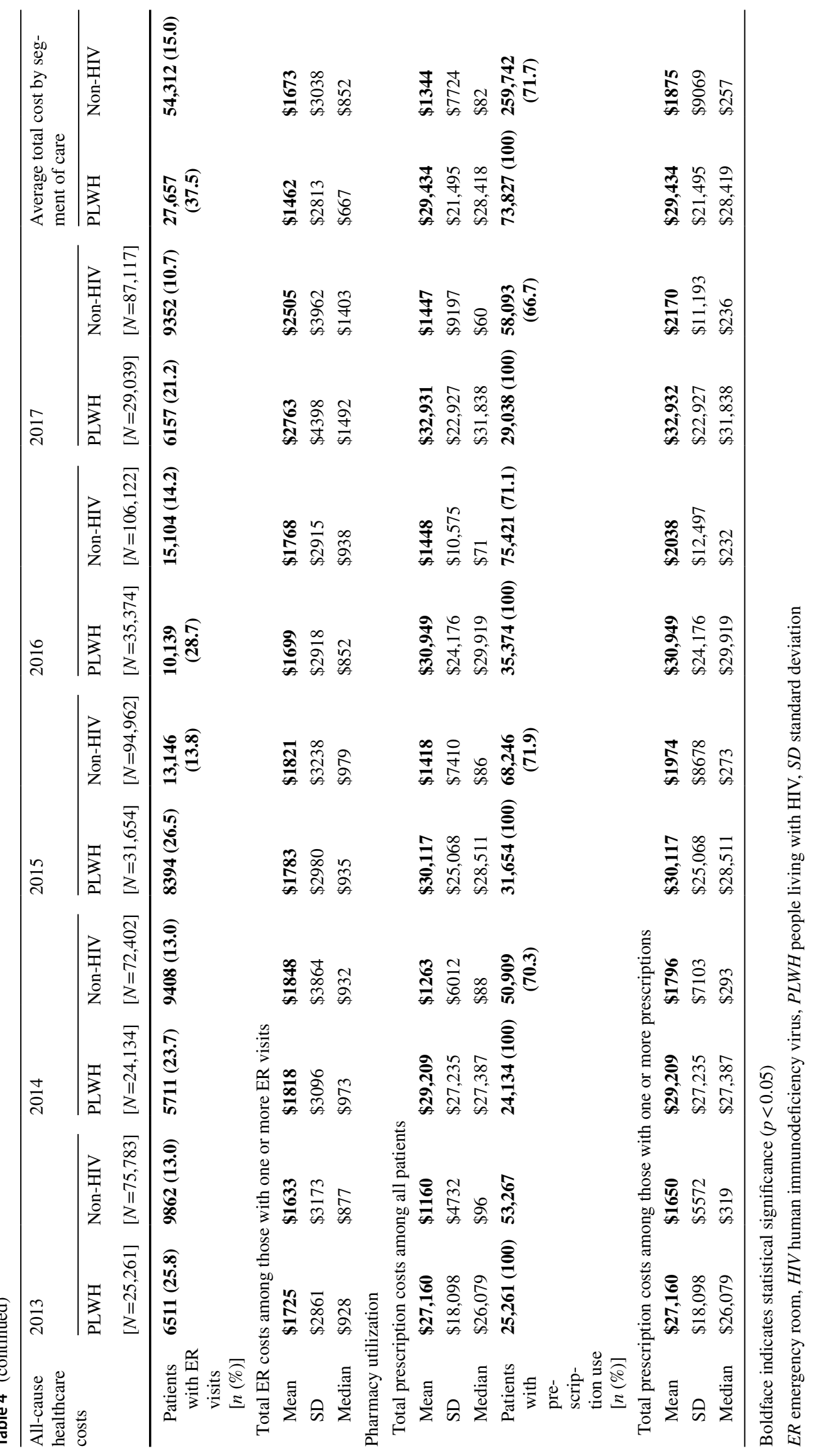


it is crucial to encourage prevention and screening for HIV, along with early initiation of ART when appropriate.

Acknowledgements The authors would like to thank Yi-Chien Lee for statistical support.

Author contributions JC: Data interpretation and manuscript review. ACB: Concept and design; data interpretation; manuscript review. YD: Concept and design; data interpretation; manuscript development. RLW: Concept and design; data interpretation; manuscript review. DMH: Concept and design; data interpretation; manuscript development; manuscript review. All authors contrib-uted to reviewing the manuscript, gave final approval of the version to be published, and agree to be accountable for all aspects of the work in ensuring that questions related to the accuracy or integrity of any part of the work are appropriately investigated and resolved.

Data Availability Statement The data used for this study are proprietary and were used under license for the current study and are therefore not publicly available.

\section{Compliance with ethical standards}

Funding This study was funded by Gilead Sciences.

Conflict of interest JC is supported by a research grant from the Drug Information Association of Europe, a non-profit association, and has served as a consultant for Gilead Sciences. AB was an employee of Gilead Sciences during the conduct of this study. DMH and RLW are employees of IQVIA, and YD was an employee of IQVIA during the conduct of this study.

Open Access This article is licensed under a Creative Commons Attribution-NonCommercial 4.0 International License, which permits any non-commercial use, sharing, adaptation, distribution and reproduction in any medium or format, as long as you give appropriate credit to the original author(s) and the source, provide a link to the Creative Commons licence, and indicate if changes were made. The images or other third party material in this article are included in the article's Creative Commons licence, unless indicated otherwise in a credit line to the material. If material is not included in the article's Creative Commons licence and your intended use is not permitted by statutory regulation or exceeds the permitted use, you will need to obtain permission directly from the copyright holder.To view a copy of this licence, visit http://creativecommons.org/licenses/by-nc/4.0/.

\section{References}

1. Centers for Disease Control and Prevention. HIV in the United States: At a glance. https://www.cdc.gov/hiv/statistics/overview/ ataglance.html. Accessed 11 Mar 2019.

2. Song R, Hall HI, Green TA, et al. Using CD4 data to estimate HIV incidence, prevalence, and percent of undiagnosed infections in the United States. J Acquir Immune Defic Syndr. 2017;74(1):3-9.
3. Hall HI, Song R, Tang T, et al. HIV trends in the United States: diagnoses and estimated incidence. JMIR Public Health Surveill. 2017;3(1):e8.

4. Satcher JA, Song R, Hall HI. Estimated HIV incidence, prevalence, and undiagnosed infections in US States and Washington, DC, 2010-2014. J Acquir Immune DeficSyndr. 2017;76(2):116-22.

5. Ritchwood TD, Bishu KG, Egede LE. Trends in healthcare expenditure among people living with HIV/AIDS in the United States: evidence from 10 years of nationally representative data. Int J Equity Health. 2017;16:188.

6. Cooke CE, Lee HY, Xing S. Adherence to antiretroviral therapy in managed care members in the United States: a retrospective claims analysis. J Manag Care Pharm. 2014;20:86-92.

7. Schackman BR, Fleishman JA, Su AE, et al. The lifetime medical cost savings from preventing HIV in the United States. Med Care. 2015;53(4):293-301.

8. Fleishman JA, Yehia BR, Moore RD, et al. The economic burden of late entry into medical care for patients with HIV infection. Med Care. 2010;48(12):1071-107.

9. Gebo KA, Fleishman JA, Conviser R, et al. HIV Research Network. Contemporary costs of HIV healthcare in the HAART era. AIDS. 2010;24(17):2705-15.

10. HIV Surveillance Report. Centers for Disease Control and Statistics. https://www.cdc.gov/hiv/statistics/overview/index.html. Accessed 5 Jun 2019.

11. US Bureau of Labor Statistics. Consumer Price Index. Washington, DC. 2019. https://www.bls.gov/cpi/home.htm. Accessed 5 Jun 2019.

12. Andrews DWK, Buchinsky M. A three-step method for choosing the number of bootstrap repetitions. Econometrica. 2000;68(1):23-51.

13. Farnham PG, Holtgrave DR, Gopalappa C, et al. Lifetime costs and quality-adjusted life years saved from HIV prevention in the test and treat era. J Acquir Immune Defic Syndr. 2013;64:e15-8.

14. Kangethe A, Polson M, Lord TC, et al. Real-World Health Plan Data Analysis: key trends in medication adherence and overall costs in patients with HIV. J Manag Care Spec Pharm. 2019;25(1):88-93.

15. UNAIDS. People aged $50+$ living with HIV. http://aidsinfo.unaid s.org/. Accessed 5 Jun 2019.

16. Wong C, Gange SJ, Moore RD, et al. Multimorbidity among persons living with human immunodeficiency virus in the United States. Clin Infect Dis. 2018;66(6):1230-8.

17. Gallant J, Hsue PY, Shreay S, et al. Comorbidities among U.S. patients with prevalent HIV infection-A trend analysis. J Infect Dis. 2017;19(12):1525-33.

18. Cole MB, Galárraga O, Rahman M, et al. Trends in comorbid conditions among Medicaid enrollees with HIV. Open Forum Infect Dis. 2019;6(4):ofz124.

19. Cohen MS, Chen YQ, McCauley M, et al. Antiretroviral therapy for the prevention of HIV-1 transmission. N Engl J Med. 2016;375:830-9.

20. Lin F, Farnham PG, Shrestha RK, et al. Cost effectiveness of HIV prevention interventions in the US. Am J Prev Med. 2016;50(6):699-708. 